\title{
Observation of Equilibrium, Nanometer-Sized Clusters of Silver Iodide in Aqueous Solutions
}

\author{
Ivana Lj. Mladenovic, ${ }^{\dagger}$ Willem K. Kegel, ${ }^{*}, \dagger$ Paul Bomans, ${ }^{\ddagger}$ and Peter M. Frederik ${ }^{\star}$ \\ Van't Hoff Laboratory for Physical and Colloid Chemistry, Debye Research Institute, Utrecht University, \\ Padualaan 8, 3584 CH Utrecht, The Netherlands, and EM Unit, Department of Pathology, University of \\ Maastricht, P.O. Box 616, 6200 MD Maastricht, The Netherlands
}

Received: November 6, 2002; In Final Form: April 15, 2003

\begin{abstract}
Thermodynamically stable clusters containing up to 10 or more silver iodide pairs have been observed in aqueous electrolyte solutions using ultracentrifugation and cryoelectron microscopy. These clusters are in equilibrium with an excess phase of solid AgI. We measured the size distribution of the clusters by their size-dependent shift of the exciton peak at visible wavelengths and show that their statistical weight is determined by their interfacial free energy. We argue that comparable equilibrium cluster distributions, but with varying widths, must be present in all insoluble salts in contact with electrolytes that contain adsorbing potential determining ions.
\end{abstract}

\section{Introduction}

It has been known for quite a while that the salt $\mathrm{AgI}$ is hardly soluble in water (solubility product $K_{\mathrm{sp}}=8.49 \times 10^{-17} \mathrm{~mol} /$ $\left.\mathrm{L}^{1}\right)$. However, when excess silver or iodide ions are added, the solubility increases dramatically, as was (probably) first studied by Hellwig as early as $1900 .^{2}$ Iodide is more effective in solubilizing AgI than silver. ${ }^{3}$ The increase in solubility is generally attributed to the formation of complexes. More than half a century later King and co-workers ${ }^{4}$ concluded that in silver iodide solutions with excess iodide, several complexes must be present containing more than a single silver atom (dubbed "polymeric species" by the authors). This conclusion was based on measured deviations from the Lambert-Beer law, i.e., a nonlinear relation between optical absorption and silver concentration in the kind of systems described above. However, complexes containing more than a single silver atom in systems with excess iodide have only been observed in acetone/water mixtures $^{5}$ and in nonaqueous solvents such as DMF. ${ }^{6}$ In the first case, X-ray scattering in combination with ultracentrifugation measurements points to the complex ion $\operatorname{Ag}_{4} \mathrm{I}_{6}{ }^{2-}$, and in the latter case, indications of the existence of complexes containing up to six silver atoms were found using potentiometric measurements.

Here we show that in aqueous solutions containing excess iodide, complexes even larger than those found in other solvents exist (up to the equivalent of $10 \mathrm{AgI}$ pairs and more). The relative concentrations of these complexes decrease exponentially with their surface area, in agreement with a simple thermodynamic model.

\section{Experimental Section}

Materials. Samples were prepared using distilled water. All chemicals (pure grade) were used without further purification.

Spectrophotometry. Optical absorption spectra were recorded using a Cary 1E UV-vis spectrophotometer. The

\footnotetext{
* To whom correspondence should be addressed. E-mail: W.K.Kegel@chem.uu.nl.

$\dagger$ Utrecht University.

$\leftarrow$ University of Maastricht.
}

temperature of the solutions was maintained at $25 \pm 0.1^{\circ} \mathrm{C}$. Cells with a $1 \mathrm{~cm}$ path length were used.

Mass Determinations. The buoyant masses of the complexes were determined in a Beckmann XL-I analytical ultracentrifuge. The ultracentrifuge contains a spectrophotometer, and the equilibrium optical absorption profile was measured at several wavelengths. It usually took between 7 and 10 days before equilibrium was established.

Cryoelectron Microscopy (Cryo-TEM). A thin film (50$200 \mathrm{~nm}$ ) was prepared in a Vitrobot $^{7}$ at $25^{\circ} \mathrm{C}$ and a relative humidity of $>95 \%$. This film was vitrified by shooting it into liquid ethane, transferred to a FEI Tecnai G2 F 30 Polara microscope, and observed at $\sim 13 \mathrm{~K}$ and $200 \mathrm{kV}$. Micrographs were recorded under low-dose conditions.

\section{Results and Discussion}

Optical absorption spectra of a system containing $2 \mathrm{M} \mathrm{KI}$ and several amounts of added silver ions are shown in Figure 1a. It can be seen from this figure that when the amount of silver in the system is increased, a clear red shift occurs: absorption takes place at increasingly longer wavelengths. This red shift may occur due to several reasons. As mentioned in the Introduction, there is general agreement that in these kind of solutions various equilibria exist, generally written as

$$
n \mathrm{Ag}^{+}+(n+m) \mathrm{I}^{-} \rightleftarrows \operatorname{Ag}_{n} \mathrm{I}_{n+m}{ }^{m-}
$$

where $\mathrm{Ag}^{+}$and $\mathrm{I}^{-}$are the silver and iodide ions, respectively, and $\operatorname{Ag}_{n} \mathrm{I}_{n+m}{ }^{m-}$ is a cluster containing $n$ silver iodide pairs and $m$ excess iodide atoms. When the (total) silver concentration is increased, the concentration of $\operatorname{Ag}_{n} \mathrm{I}_{n+m}{ }^{m-}$ clusters obviously rises. It will rise linearly with the (total) silver concentration if only a single species is present, but it will change nonlinearly if other complexes appear. The first scenario leads to a constant absorption ratio between two solutions containing different amounts of silver (a manifestation of the Lambert-Beer law), while the second scenario will show up in an absorption ratio that is not constant. As can be seen in Figure 1a (inset), clearly, 

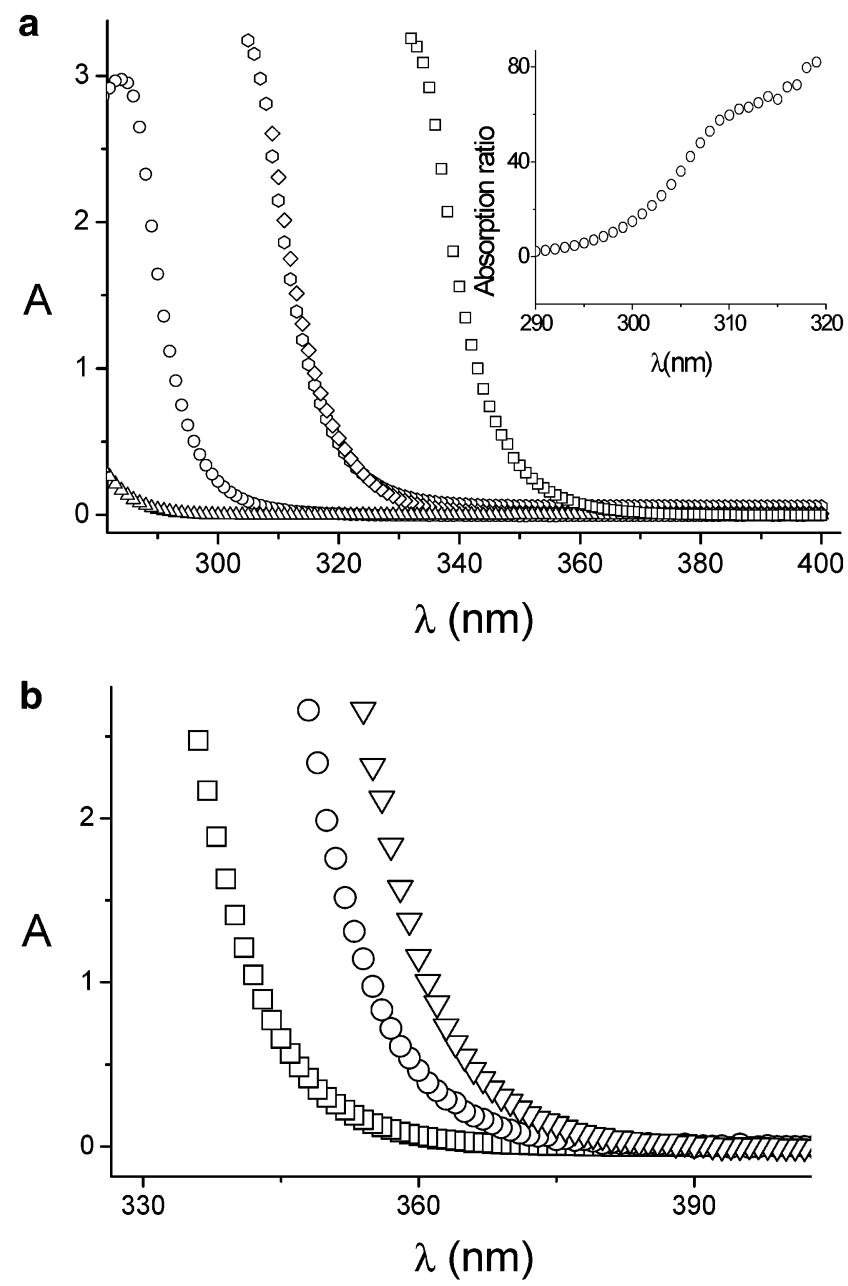

Figure 1. (a) Optical absorption of solutions containing $2 \mathrm{M} \mathrm{KI}$ and $0.5 \mathrm{M} \mathrm{AgClO}_{4}(\square), 0.021 \mathrm{M} \mathrm{AgNO}_{3}(\diamond), 0.021 \mathrm{M} \mathrm{AgClO}_{4}(\diamond), 3.33$ $\times 10^{-3} \mathrm{M} \mathrm{AgNO}_{3}(\mathrm{O})$, and $1 \times 10^{-4} \mathrm{M} \mathrm{AgNO}_{3}(\triangle)$. These absorptions were measured relative to those of a system without silver. Sodium thiosulfate $\left(8 \times 10^{-4} \mathrm{M}\right)$ was present in the systems to avoid formation of $\mathrm{I}_{3}{ }^{-4}$. The inset shows the ratio between absorption of 0.021 and 3.33 $\times 10^{-3} \mathrm{M} \mathrm{Ag}$ in the system. (b) Like Figure 1a, but now with a constant ratio of silver to iodide (1:4) and different (total) iodide concentrations of $2(\square), 3(\bigcirc)$, and $5 \mathrm{M}(\nabla)$. In these systems, an excess phase of solid $\mathrm{AgI}$ is present. The absorption data were taken from the liquid phases of the systems after removal of the solid by centrifugation.

the second scenario applies: the observed red shift occurs due to the appearance of other clusters when the silver concentration increases.

The largest red shift occurs when an excess phase of solid silver iodide is present. It becomes even larger upon when the iodide concentration is increased, as shown in Figure 1b. We will now address the question of the sizes of the complexes in these systems. In nanosized semiconductor particles, the band gap energy of an electron-hole pair depends on the size of the particles (see, for example, ref 8). In the strongly confined limit, where the Bohr radius is much larger than the particle size, the bulk exciton peak of a spherical particle of radius $R$ is centered at a wavelength $\lambda$, given by

$$
\frac{1}{\lambda}=\frac{1}{\lambda_{0}}+\frac{\alpha}{R^{2}}
$$

where $\lambda_{0}$ is the wavelength where the bulk exciton peak is observed and the constant $\alpha$ depends on the Bohr radius and the high-frequency dielectric constant of the particles (see again

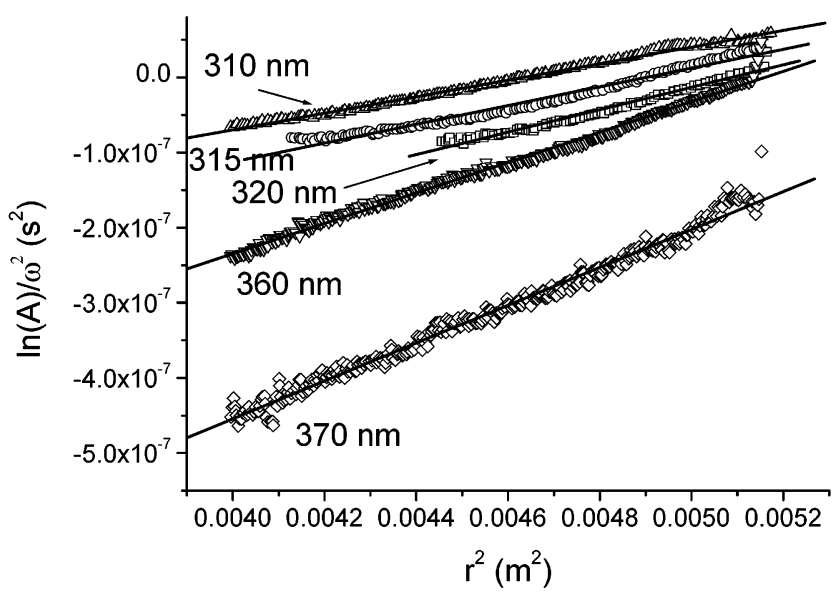

Figure 2. Equilibrium optical absorption profiles of systems with several iodide concentrations and a 4:1 I:Ag ratio in an ultracentrifuge, plotted as $\ln A / \omega^{2}$ vs $r^{2}$ (see eq 3 and the discussion following it in the text). The different wavelengths at which the profiles were taken are indicated in the figure. The buoyant masses, $m_{\mathrm{b}}$, of the particles that follow from the slopes of the curves are as follows: $m_{\mathrm{b}}=8.9 \times 10^{-25}$ $\mathrm{kg}(310 \mathrm{~nm}, 0.57 \mathrm{M}$ iodide $), m_{\mathrm{b}}=9.8 \times 10^{-25} \mathrm{~kg}(315 \mathrm{~nm}, 0.57 \mathrm{M}$ iodide), $m_{\mathrm{b}}=13 \times 10^{-25} \mathrm{~kg}(320 \mathrm{~nm}, 0.57 \mathrm{M}$ iodide $), m_{\mathrm{b}}=17 \times$ $10^{-25} \mathrm{~kg}(350 \mathrm{~nm}, 2 \mathrm{M}$ iodide $)$, and $m_{\mathrm{b}}=20 \times 10^{-25} \mathrm{~kg}(370 \mathrm{~nm}, 5$ $\mathrm{M}$ iodide). We transform the observed buoyant masses to equivalent masses of an AgI pair, $n$, by writing $m_{\mathrm{b}}=n\left(m_{\mathrm{AgI}}-v \rho_{\mathrm{s}}\right)$. In this equation, $m_{\mathrm{AgI}}\left(=3.9 \times 10^{-25} \mathrm{~kg}\right)$ is the mass of an AgI pair, $v(=0.0699$ $\mathrm{nm}^{3}$ ) is the volume of AgI in the thermodynamically stable solid $\beta$ (wurtzite) phase, and $\rho_{\mathrm{s}}$ is the (mass) density of the solvent water with salt. The value of $v$ that we took assumes that the clusters have the same structure as the macroscopic solid AgI. In practice, we expect different structures: as long as they are small, the structure will be adjusted to minimize the interfacial free energy. However, the volumes of AgI in the different solid phases vary less than $10 \% ;{ }^{11}$ even the $v$ value of $0.075 \mathrm{~nm}^{3}$ deduced from the $\mathrm{Ag}_{4} \mathrm{I}_{6}{ }^{2-5}$ cluster is only a few percent different from the one that we employ. Moreover, the value of $n$ is quite insensitive for the choice of $v$ : in this case, the value of $v \rho_{\mathrm{s}}$ $\left(\approx 7 \times 10^{-26} \mathrm{~kg}\right)$ being only $\sim 18 \%$ of the value of $m_{\mathrm{AgI}}$. This leads to $n$ values of $2.8(310 \mathrm{~nm}), 3.1(315 \mathrm{~nm}), 4.1(320 \mathrm{~nm}), 5.3(350 \mathrm{~nm})$, and $6.2(370 \mathrm{~nm})$.

ref 8). In the case of very small particles, in particular when they approach a molecular size, eq 2 is expected to break down. However, several observations of complexes of molecular size AgI $(n=2-4)$ point to a size dependence of the exciton absorption that is at least qualitatively similar to eq 2 (see, for example, refs 9 and 10). Thus, the red shift as observed in Figure 1 is caused by the appearance of larger and larger complexes in the system. This was independently corroborated by ultracentrifugation (UC) measurements, where we measured the optical absorption profile as a function of wavelength. The concentration as a function of radial position $r, c(r)$, of a single species of buoyant mass $m_{\mathrm{b}}$ is given by

$$
c(r)=c\left(r_{0}\right) \exp \left[\frac{m_{\mathrm{b}} \omega^{2}\left(r^{2}-r_{0}^{2}\right)}{2 k T}\right]
$$

where $r_{0}$ is a reference radial position (usually the position of the liquid-air meniscus of the sample), $\omega$ is the rotational velocity, and $k$ and $T$ are Boltzmann's constant and the absolute temperature, respectively. Equation 3 applies if the species behave ideally, i.e., if interactions between them are negligible. As will be shown later (cf. Figure 4), the concentrations of the species are small enough for that to be the case. If several different species are present, they will, depending on their size, absorb at different wavelengths. The optical absorption profile $A(r)=\epsilon l c(r)$, where $c(r)$ is given by eq 3 and $\epsilon$ and $l$ are the 


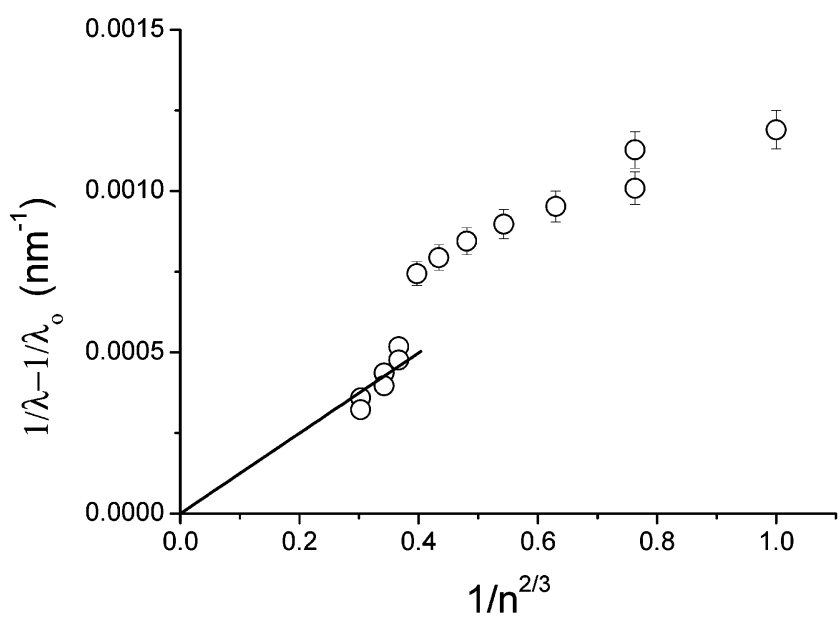

Figure 3. Plot of $1 / \lambda-1 / \lambda_{0}$ vs $n^{-2 / 3}$, where $n$ is the equivalent mass of a cluster in units of AgI pairs. The values of $n$ were calculated from the buoyant masses as indicated below Figure 2. $\lambda$ is the wavelength at which a corresponding buoyant mass was observed, as indicated in Figure 2. $\lambda_{0}(=420 \mathrm{~nm})$ is the value of the wavelength where the bulk exciton absorption of $\mathrm{AgI}$ is centered. ${ }^{20}$ The (six) largest $n$ values correspond to systems with 2, 3, and $5 \mathrm{M}$ iodide. The uncertainties in these values are smaller than the symbol sizes. The other points were obtained from systems with $0.57 \mathrm{M}$ iodide. No systematic difference was found between these systems. According to eq 3, the points should lie on a straight line that goes through the origin. This is only the case for the largest $(n>4)$ clusters. The slope of this line gives an $\alpha$ of $(0.82 \pm 0.03) \times 10^{-4} \mathrm{~nm}$

(molar) extinction coefficient and the optical path length, respectively. This profile will depend on wavelength, because different wavelengths will pick up different sizes and thus different buoyant masses ( $m_{\mathrm{b}}$ in eq 3 ). As can be seen in Figure 2, this is exactly what happens: the larger the wavelength, the larger the buoyant mass of the particles. The (real) masses of the particles that can be extracted from Figure 2 correspond to an equivalent number of $\mathrm{AgI}$ pairs in the cluster between 2.8 $(310 \mathrm{~nm})$ and $6.2(370 \mathrm{~nm})$, assuming that clusters of size $n$ only absorb at a single wavelength. This assumption is quite plausible, as clusters significantly absorbing at more than a single wavelength would show up in systematic deviations from straight lines in Figure 2. This clearly does not occur. At wavelengths above $370 \mathrm{~nm}$, the absorption intensity became too small and noisy for extraction of reliable masses. This is due to a combination of a small optical path length of the cell that was used in the UC measurements and the limited sensitivity of the optical detector of the UC: the systems still absorb significantly at wavelengths beyond $370 \mathrm{~nm}$, pointing to the presence of clusters for which $n>6.2$.

To extract the size distribution of the complexes from the optical absorption spectra, we need a quantitative relation between the size of a cluster and the wavelength where it absorbs. According to eq 2 , plotting $1 / \lambda-1 / \lambda_{0}$ versus $n^{-2 / 3} \propto$ $R^{-2}$ should give a straight line that goes through the origin. As shown in Figure 3, where we plotted these quantities for a variety of systems, this is only the case for the largest $(n>4)$ clusters. The value of the constant $\alpha\left[(0.82 \pm 0.03) \times 10^{-4}\right.$ $\mathrm{nm}$ ] that was extracted from Figure 3 is roughly 1 order of magnitude smaller than the theoretical value of $\alpha\left(=\pi e^{2} a_{\mathrm{B}} /\right.$ $\left.8 c h \epsilon_{\mathrm{r}} \epsilon_{0} \approx 1.2 \times 10^{-3} \mathrm{~nm}\right)$. In this equation, $e$ is the electron charge and $c, h$, and $\epsilon_{0}$ are the speed of light (in vacuum), Planck's constant, and the vacuum permittivity, respectively. The Bohr radius $a_{\mathrm{B}}=1.1 \mathrm{~nm}$ for $\mathrm{AgI}$, and the high-frequency dielectric constant $\epsilon_{\mathrm{r}}=4.9 .{ }^{11}$ The difference between the theoretical and experimental values of $\alpha$ is hardly surprising, considering the small size of the clusters. Note that any empirical function that describes the relation between absorption wavelength and particle size in Figure 3 will serve our purpose equally well. We will further use eq 2 to extract the number of $\mathrm{AgI}$ pairs from the wavelength

$$
n(\lambda)=\frac{4 \pi}{3 v} \alpha^{3 / 2}\left(\frac{1}{\lambda}-\frac{1}{\lambda_{0}}\right)^{-3 / 2}
$$

where we used the geometrical relation $4 / 3 \pi R^{3}=n v$ with $v=$ $0.0699 \mathrm{~nm}^{3}$ for the volume of $\mathrm{AgI}$ in the thermodynamically stable solid $\beta$ (wurtzite) phase. In deriving eq 4 , we assumed that the clusters are spherical. In general, different cluster shapes will lead to different values of the geometrical factor $4 \pi / 3$ in eq 4.

We will further concentrate on systems with a constant ratio of silver and iodide (1:4), but varying iodide concentrations, as in Figure 1b. In all these systems, a macroscopic phase of (excess) solid silver iodide is present. We have carefully checked if the systems are in thermodynamic equilibrium; i.e., we performed temperature cycles and added the components in a different order. In all cases, we find indistinguishable absorption spectra. We therefore conclude that the clusters that we find are in thermodynamic equilibrium with an excess solid phase. The distribution of clusters can be predicted by thermodynamics. In Appendix 1, we analyze the equilibrium

$$
n \mathrm{AgI}(\mathrm{s})+m \mathrm{I}^{-} \rightleftarrows \operatorname{Ag}_{n} \mathrm{I}_{n+m}{ }^{m-}
$$

where $\operatorname{AgI}(\mathrm{s})$ is the (macroscopic) solid, undissolved aggregate of silver iodide. This analysis leads to the size distribution of clusters

$$
c_{n}=b \mathrm{e}^{-a n^{2 / 3} / k T}
$$

where $c_{n}$ is the (molar) concentration of clusters containing $n$ AgI pairs and, in principle, every possible number of excess iodide ions. We expect that the dependence on $n$ of the first term on the right-hand side of eq 6 is weak compared to the exponential dependence in the second term. The term in the exponent of eq 6 contains the interfacial free energy of the cluster. In the case of spherical clusters with a radius $R$, we have $a n^{2 / 3}=4 \pi R^{2} \gamma$, where $\gamma$ is the interaction free energy per unit area. If clusters are large (say $n>100$ ), $\gamma$ can be considered as the macroscopic interfacial tension between excess solid AgI and the electrolyte solution. In that case, $a=4 \pi(3 v / 4 \pi)^{2 / 3} \gamma$, where $v$ is the volume of an AgI pair inside bulk solid AgI. For smaller clusters, $\gamma$ can no longer be regarded as a macroscopic quantity. However, we do not expect its value to be much different from the interfacial tension of the macroscopic solidelectrolyte interface. The size distribution may now be deduced from the absorption spectrum: the relation between $n$ and $\lambda$ is given by eq 4 and the relation between $c_{n}$ and optical absorption by the Lambert-Beer law.

In Figure 4, we plotted the (logarithm of the) optical absorption as a function of $n^{2 / 3}$ for three different systems with a constant ratio of silver and iodide (1:4), but varying iodide concentrations of 2,3 , and $5 \mathrm{M}$. Obviously, the higher the iodide concentration, the larger the clusters in the system become. In the case of the system with $5 \mathrm{M}$ iodide, in the tail of the size distribution, clusters with $n=10$ are observed, as can be seen in Figure 4. Clusters with $n=5-10$ should be on the order of $1 \mathrm{~nm}$ in size. Figure 5 shows a cryoelectron microscopy picture of the system with $5 \mathrm{M}$ iodide, from which it is obvious that, indeed, clusters on the order of this size exist. As far as we are 

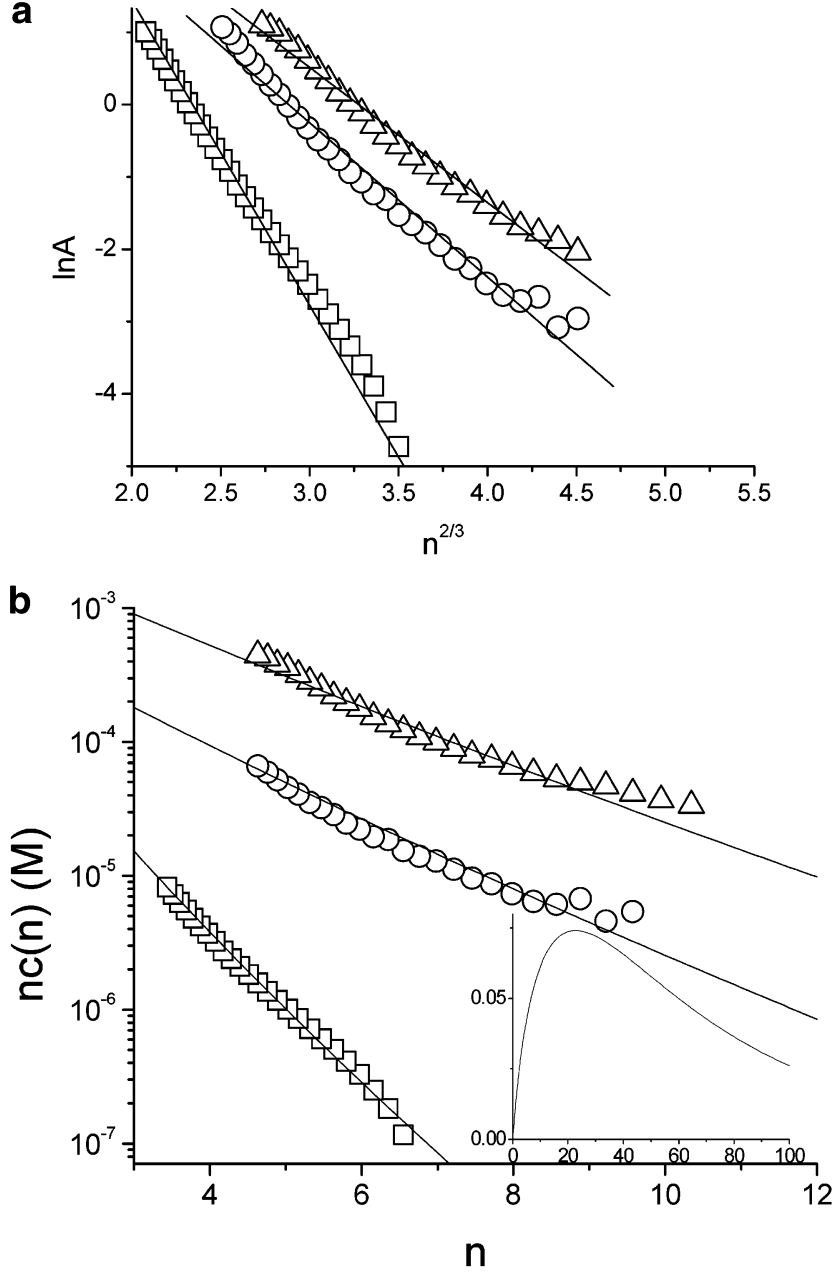

Figure 4. (a) Same as Figure $1 \mathrm{~b}$, but with the logarithm of the absorption plotted vs $n^{2 / 3}$, where $n$ was calculated using eq 4 with an $\alpha$ of $(0.82 \pm 0.03) \times 10^{-4} \mathrm{~nm}$. As explained in the text, the slopes of the lines in Figure 4 equal $-a / k T$ (see eq 6), whereas their intercepts are given by $\ln (\epsilon l b)$. For the systems with 2,3 , and $5 \mathrm{M}$ iodide, the slopes are $-3.78,-2.13$, and -1.88 and the intercepts are $8.78,6.14$, and 6.11, respectively. (b). Concentration of AgI in clusters of size $n$, $n c_{n}$, with $c_{n}$ given by eq 6 . The values of $b$ were determined as explained in Appendix 2. They are $0.014,0.0051$, and $0.015 \mathrm{M}$ for the systems containing 2, 3, and $5 \mathrm{M}$ iodide, respectively. In the inset, we show the size distribution for $a / k T=0.18$, i.e., one-tenth of the value of the $5 \mathrm{M}$ system, and $b=0.015 \mathrm{M}$.

aware, these are the smallest AgI "particles" that have ever been observed by this technique. Since these sizes are on the order of the resolution of the microscopy technique, we could not extract reliable size distributions from Figure 5.

From Figure 4, we conclude that our measured cluster distributions are fairly well described by the exponential form as predicted by theory. The deviations from the straight lines in this figure might be due to a weak $n$ dependence of the prefactor $b$ in eq 6 , but may also be caused by a (weak) $n$ (or wavelength)-dependent molar extinction coefficient of the clusters. The slopes of the lines in Figure 4 include the constant $a$ (see eq 6 ), whereas their intercepts are given by $\ln (\epsilon l b)$. The values of $a / k T$ are $3.78,2.13$, and 1.88 , corresponding to values of $\gamma$ of $18.9,10.7$, and $9.3 \mathrm{mN} / \mathrm{m}$ for the systems containing 2, 3 , and $5 \mathrm{M}$ iodide, respectively. The values of $\gamma$ are expected to be comparable to the interfacial tension of the macroscopic AgI-electrolyte interface. The interfacial tension of the AgIwater interface, without adsorbed iodide, is on the order of 100 $\mathrm{mN} / \mathrm{m}$, based on the comparable interfacial tensions of $\mathrm{AgBr}$

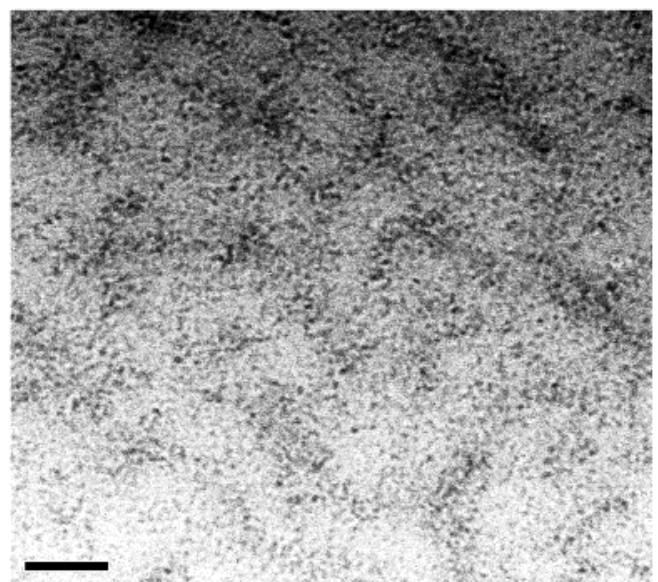

Figure 5. Cryoelectron microscopy image (see the Experimental Section) of the system with $5 \mathrm{M}$ iodide. The micrograph shows a population of small grains, not present in the control sample (system without silver, image not shown). The bar represents $20 \mathrm{~nm}$.

and $\mathrm{AgCl}$ (see, for example, ref 12). Thus, addition of iodide reduces the interfacial tension by 1 order of magnitude. This reduction must be caused by adsorption of iodide at the AgIwater interface. Note that surfactants reduce the interfacial tension of the air-water interface by the same order of magnitude. ${ }^{13}$ In water-surfactant systems, micelles are formed. These micelles are quite monodisperse. Here we find the analogue of micelles built from inorganic material, but these have a continuously decreasing size distribution. This is because in the case of micelles, their equilibrium size is determined by the geometry of the surfactant molecules, ${ }^{13}$ while here, it is purely the interfacial free energy that determines the (Boltzmann) weight of the species.

In Appendix 2, we describe how the values of $b$ in eq 6 were obtained. With these values, the cluster size distribution, eq 6 , is now fully determined. In Figure $4 \mathrm{~b}$, we plotted the concentration of AgI pairs in clusters of size $n, n c_{n}$. As can be seen in this figure, the system with the highest iodide concentration contains a significant fraction of silver in clusters in which $n \geq$ 10. Thermodynamically stable clusters of AgI of this size have never been seen before, not in aqueous solutions or in other solvents. Other insoluble salts in contact with the electrolyte that contains adsorbing potential-determining ions are expected to behave similarly. However, the sizes of the clusters being formed sensitively depend on the value of $a$ in eq 6 . This value is in turn determined by the adsorption density of the ions at the solid-electrolyte interface. Thus, clusters with sizes similar to the ones found here are expected in systems where potentialdetermining ions adsorb strongly, like iodide on $\mathrm{AgI}{ }^{3}$ In principle, it might be possible to reduce the lowest interfacial tension that we find by an additional 1 order of magnitude. This may be accomplished by adding material that adsorbs more strongly than iodide. However, the adsorption process should still be reversible so that exchange of matter between the clusters is possible; this is a necessary condition for thermodynamic equilibrium. As shown in the inset of Figure $4 b$, with such a smaller value of $a$ in eq 6 , the size distribution $n c_{n}$ will first increase with $n$, peak at $n \approx 20$, and subsequently slowly fall off at larger $n$ values, leaving a significant fraction of $\mathrm{AgI}$ in clusters with $n>100$. This scenario is comparable to the one discussed by Overbeek, ${ }^{14}$ who argued that insoluble solid materials may emulsify spontaneously by reducing the interfacial tension between the solid-solute interface. 


\section{Concluding Remarks}

We showed that silver iodide in the presence of excess iodide spontaneously forms clusters up to $\sim 1 \mathrm{~nm}$ in size. These clusters are largest (on average) if excess solid silver iodide is present. The statistical weight of a cluster in the size distribution is exponentially decreasing with its interfacial area. We have already mentioned the analogy with micelles. In fact, there are more situations where two or more immiscible materials may be interdispersed on nanometer length scales and be thermodynamically stable, for example, microemulsions (see, for example, ref 15). These are mixtures of oil and water stabilized by surfactants. But there also are examples of thermodynamically stable dispersions of large (solid) clusters or nanosized particles in a solvent. In the presence of acids, clusters containing up to 36 metal atoms spontaneously (and reversibly!) form in aqueous solutions of molybdenum and tungsten oxide (see, for example, ref 16). In certain mixtures of iron oxide, ${ }^{17}$ but now at high $\mathrm{pH}$ and in an aggregated state, particles several nanometers in size are found to form reversibly. In the first example, contrary to what we find, surface effects appear not to play a role. But in the second example, it might be that adsorbed $\mathrm{OH}^{-}$ions stabilize the particles. Still another example is thiol-capped gold nanoparticles in nonaqueous solvents, ${ }^{18}$ but it is not clear whether these systems are reversible. We showed that insoluble salts may also (partly) emulsify spontaneously; we are currently systematically investigating the effects of species other than iodide that adsorb at the AgI-electrolyte interface.

Acknowledgment. We thank Jan Groenewold for many suggestions and discussions and Daniël Vanmaekelbergh and Andries Meijerink for kindly sharing their knowledge of the optics of quantum dots. Daniël Vanmaekelbergh is also thanked for useful comments on the manuscript. This work was supported by CW/NWO.

\section{Appendix 1}

Multichemical Equilibrium and Cluster Size Distribution. Clusters of silver iodide are in equilibrium with a solid phase of AgI described by

$$
n \mathrm{AgI}(\mathrm{s})+m \mathrm{I}^{-} \rightleftarrows \operatorname{Ag}_{n} \mathrm{I}_{n+m}{ }^{m-}
$$

where $\operatorname{AgI}(\mathrm{s})$ is the (macroscopic) solid, undissolved aggregate of silver iodide, $\mathrm{I}^{-}$is the iodide ion, and $\operatorname{Ag}_{n} \mathrm{I}_{n+m}{ }^{m-}$ is a cluster containing $n$ silver iodide pairs and $m$ excess iodide atoms.

Thermodynamic equilibrium implies that $\sum_{\mathrm{i}} v_{\mathrm{i}} \mu_{\mathrm{i}}=0$, where $\nu_{\mathrm{i}}$ and $\mu_{\mathrm{i}}$ are the stoichiometric coefficients and the chemical potentials of components $i$, respectively. The chemical potentials of the soluble species depend on concentration. If $\mathrm{i}=\mathrm{I}^{-}$, we write the chemical potential as $\mu_{\mathrm{I}^{-}}=\mu_{\mathrm{I}^{-}}^{0}+k T \ln \left(f_{-} c_{\mathrm{I}^{-}}\right)$, where $\mu_{\mathrm{i}}^{0}$ is the standard chemical potential, $c_{\mathrm{I}^{-}}$the concentration of $\mathrm{I}^{-}$relative to the "standard concentration" $\left(c^{0}=1 \mathrm{M}\right)$, and $f_{-}$ is the activity coefficient (fugacity) of a negatively charged univalent electrolyte. Similarly, for $\operatorname{Ag}_{n} \mathrm{I}_{n+m}{ }^{m-}$, we write the chemical potential as

$$
\mu_{n, m}=\mu_{n, m}^{0}+k T \ln x_{n, m}
$$

where the subscript $n, m$ refers to the cluster $\operatorname{Ag}_{n} \mathrm{I}_{n+m}{ }^{m-}$. The logarithmic term in the above equation now contains the mole fraction $x_{n, m}$ instead of the (molar) concentration. The reason for this choice is that we take the (excess) solid phase, where $x=1$, as the standard state that defines (part of) $\mu_{n, m}^{0}$. As only (very) small concentrations of the species are expected, we neglect deviations from ideal behavior of the clusters. Clusters with an $n, m$ composition contain $n$ AgI pairs as well as $m$ excess iodide ions. As opposed to the situation in the excess solid, the clusters are surrounded by an electrolyte solution rather than being part of a lattice. This gives rise to an interfacial free energy term in the standard chemical potential of a cluster. We therefore write

$$
\mu_{n, m}^{0}=n \mu_{\infty}^{0}+m \mu_{\mathrm{I}^{-}}^{\text {ins }}(n)+a n^{2 / 3}
$$

This equation can be considered as the reversible work of building an $n, m$ cluster involving three steps.

Step 1 is formation of $n$ pairs of bulk AgI [i.e., (excess) solid AgI in the thermodynamic limit]. This contribution is $n \mu_{\infty}^{0}$, where $\mu_{\infty}^{0}$ is the chemical potential of an AgI pair in the bulk (this term is going to cancel the contribution from the excess solid);

Step 2 is addition of $m$ excess iodide molecules to the cluster; this contributes $m \mu_{\mathrm{I}^{-}}^{\text {ins }}(n)$, where $\mu_{\mathrm{I}^{-}}^{\text {ins }}(n)$ is the chemical potential of excess iodide inside a cluster. This quantity is expected to depend on the number of AgI pairs in a cluster. If excess iodide mixes randomly with $\mathrm{AgI}$, then $\mu_{\mathrm{I}^{-}}^{\text {ins }}(n)$ would have the same form as eq A2, where in that case $x=m /(n+m)$. However, since iodide merely adsorbs onto the clusters, we expect a more complicated dependence on $n$. We will not make an assumption for this dependence.

Step 3 is the surface term. This term takes into account the interactions of an $n, m$ cluster with the electrolyte (after being removed from the bulk excess solid). This term is the interfacial free energy of the cluster which we write as $a n^{2 / 3}$. In case of spherical clusters of radius $R$, we have $a n^{2 / 3}=4 \pi R^{2} \gamma$, where $\gamma$ is the interaction free energy per unit area. As explained in the main text, $\gamma$ can be considered the macroscopic interfacial tension between excess solid AgI and the electrolyte solution if clusters are large (say $n>100$ ).

Combining the above equations leads to the size distribution of clusters of the $n, m$ category

$$
c_{n, m}=v_{\mathrm{s}}^{-1}\left[f_{-} c_{\mathrm{I}^{-}} \mathrm{e}^{\Delta \mu_{\mathrm{I}}^{\mathrm{ins}}(n)}\right]^{m} \mathrm{e}^{-a n^{2 / 3} / k T}
$$

In this equation, $c_{n, m}\left(=x_{n, m} / v_{\mathrm{s}}\right)$ is the (molar) concentration of the clusters of the $n, m$ category, $v_{\mathrm{s}}$ is the (molar) volume of the solvent (for water, $v_{\mathrm{s}}^{-1} \approx 55.56 \mathrm{M}$ ). We have defined $\Delta \mu_{\mathrm{I}^{-}}^{\text {ins }}(n)$, which equals $\left[\mu_{\mathrm{I}^{-}}^{0}-\mu_{\mathrm{I}^{-}}^{\text {ins }}(n)\right] / k T$, as being the difference in chemical potential between ( $1 \mathrm{~mol}$ of) iodide in solution and iodide in a cluster. This term takes into account that upon formation of an $n, m$ cluster, $m$ iodide ions are transferred from solution to the solid cluster. Thus, $\Delta \mu_{\mathrm{I}^{-}}^{\text {ins }}(n)$ quantifies the fraction of iodide that adsorbs on a cluster of size $n$. We will neglect any explicit dependence of $\Delta \mu_{\mathrm{I}^{-}}^{\text {ins }}(n)$ on $n$, thereby decoupling the second and third term on the right-hand side of eq A4.

The value of $m$ equals the charge of a cluster. There are two extreme cases. If all clusters have equal charge $\langle m\rangle$, then the size distribution of clusters containing $n$ AgI pairs can be written as $c_{n}=v_{\mathrm{s}}{ }^{-1}\left(f_{-} c_{\mathrm{I}^{-}} \mathrm{e}^{\mathrm{s}^{u_{\mathrm{I}}^{-}}}\right)^{\langle m\rangle} \mathrm{e}^{-a n^{2 / 3} / k T}$. In the other extreme case, a cluster of $n$ AgI pairs may have every possible charge and one has to sum over $m$ to obtain the distribution of clusters containing $n \mathrm{AgI}$ pairs (independent of the number of iodide atoms) 


$$
c_{n}=\sum_{m=0}^{\infty} v_{\mathrm{s}}^{-1}\left(f_{-} c_{\mathrm{I}^{-}} \mathrm{e}^{\Delta \mu_{\mathrm{I}^{-}}^{\mathrm{ins}}}\right)^{m} \mathrm{e}^{-a n^{2 / 3} / k T}=\frac{v_{\mathrm{s}}^{-1}}{1-f_{-} c_{\mathrm{I}^{-}} \mathrm{e}^{\Delta \mu_{\mathrm{I}^{-}}^{\text {ins }}}} \mathrm{e}^{-a n^{2 / 3 / k T}}
$$

provided that $f_{-} c_{\mathrm{I}}^{-} \mathrm{e}^{\Delta \mu_{\mathrm{I}}^{-}}<1$ and that all values of $m$ are equally probable. These extreme cases are not very plausible; for example, experiments in DMF point to complexes containing one to three iodide atoms in species consisting of a single silver atom (see, for example, ref 6). In this example, the number of (excess) iodide atoms is not fixed, nor are all (from zero to infinity) values of $m$ allowed. On the other hand, solubility data ${ }^{4}$ point to a constant (average) charge of the clusters over a wide range of iodide concentrations. We feel that this issue has not been resolved and requires further study. We will therefore not make any assumptions and pragmatically write the size distribution as eq 6 in the main text

$$
c_{n}=b \mathrm{e}^{-a n^{2 / 3} / k T}
$$

where the "prefactor" $b$ only depends on the iodide concentration in solution and the average charge of the clusters. The dependence of $b$ on $n$ is expected to be weak compared to the exponential dependence, i.e., the second term on the right-hand side of eq A5.

\section{Appendix 2}

Data Analysis. The values of $a$ in eq A5 are obtained as described in the main text. The values of $b$ cannot simply be extracted from the absorption spectrum, since the absolute optical absorption of the clusters is not precisely known. Samples were measured relative to samples without silver, but these samples still have a composition slightly different from that of the electrolyte in which the clusters are dispersed. Therefore, we extracted the values of $b$ from solubility data of silver at different iodide concentrations, as reported in ref 4. Assuming that all silver is dispersed in the form of clusters (i.e., the number of free silver ions is much smaller than the amount of silver in the clusters), the solubility of silver is given by $c_{\mathrm{Ag}}$ $=\sum_{n=1}^{\infty} n c_{n}=b \sum_{n=1}^{\infty} n \mathrm{e}^{-a n^{2 / 3} / k T}$. In principle, the values of $b$ can now be extracted from this equation using the solubilities $c_{\mathrm{Ag}}$ as well as the values of $\sum_{n=1}^{\infty} n \mathrm{e}^{-a n^{2 / 3} / k T}$ that were calculated numerically.

The solubility data were taken from ref 4 and are described well by the function $\log \left(c_{\mathrm{Ag}}\right)=-3.91+2.97\left[\log \left(c_{\mathrm{I}^{-}}\right)\right]$. The values of $c_{\mathrm{I}^{-}}$are in fact the effective iodide concentrations in the system; we compensated for the amount of iodide that is present in the solid excess phase of AgI. Since in all cases almost all silver resides in the solid, we simply subtracted the total concentration of $\mathrm{Ag}$ in the sample from the initial iodide concentration. As a result (for the constant 1:4 ratio of silver to iodide), $c_{\mathrm{I}^{-}}=1.5,2.25$, and $3.75 \mathrm{M}$ instead of 2, 3, and $5 \mathrm{M}$, respectively, corresponding to $c_{\mathrm{Ag}}$ values of $4 \times 10^{-4}$,
$1.3 \times 10^{-3}$, and $6.0 \times 10^{-3} \mathrm{M}$, respectively. The values of $\sum_{n=1}^{\infty} n \mathrm{e}^{-a n^{2 / 3} / k T}$ are $0.029,0.26$, and 0.41 for the values of $a$ that correspond to 2,3 , and $5 \mathrm{M}$ iodide, respectively. This leads to $b$ values of $0.014,0.0051$, and $0.015 \mathrm{M}$ for the systems containing 2,3 , and $5 \mathrm{M}$, respectively. If clusters of size $n$ contain a constant number $m$ (or $\langle m\rangle$ ) adsorbed iodide ions, and if this value only weakly varies with $n$, then $b \approx$ $v_{\mathrm{s}}{ }^{-1}\left(f_{-} c_{\mathrm{I}}{ }^{-} e^{\Delta_{\mathrm{I}^{-}}^{\text {ins }}}\right)^{\langle m\rangle}$ and increases with iodide concentration. Clearly, this only happens upon increasing the total iodide concentration from 3 to $5 \mathrm{M}$, where a $b\left(c_{\mathrm{I}^{-}}=5 \mathrm{M}\right) / b\left(c_{\mathrm{I}^{-}}=3\right.$ $\mathrm{M})$ ratio of $\approx 2.9$ points to an $\langle m\rangle$ of $\approx 2.1$, consistent with a charge of the clusters being more negative than -2 as found in ref 4 using electrophoresis. We expect that the model (eq 6) breaks down for very small cluster sizes. Indeed, the summation $c_{\text {Ag }}=\sum_{n=1}^{\infty} n c_{n}$, including clusters of sizes as small as 1 , will certainly lead to errors. The system in which $c_{\mathrm{I}^{-}}=2 \mathrm{M}$ contains the largest fraction of small clusters, which may explain the unexpectedly high value of $b$ for this system.

Comparing the values of the intercepts of the curves in Figure 4 (i.e., 8.78, 6.14, and 6.11 for systems containing 2, 3, and 5 $\mathrm{M}$ iodide, respectively) with those of $b$ immediately gives an estimate of the molar extinction coefficient of the clusters: it is on the order of $10^{5} \mathrm{~L} \mathrm{~mol}^{-1} \mathrm{~cm}^{-1}$ (with our optical path length of $1 \mathrm{~cm}$ ). This is comparable to the value reported by Mulvaney ${ }^{19}\left(7.6 \times 10^{5} \mathrm{~L} \mathrm{~mol}^{-1} \mathrm{~cm}^{-1}\right.$ for his AgI particles that were $3 \mathrm{~nm}$ in diameter).

\section{References and Notes}

(1) Handbook of Chemistry and Physics, 66th ed.; CRC Press: Boca Raton, FL, 1985-1986.

(2) Hellwig, K. Z. Anorg. Chem. 1900, 25, 157.

(3) Weiser, H. B. The colloidal salts; McGraw-Hill: London, 1928.

(4) King, E. L.; Krall, H. J.; Pandow, M. L. J. Am. Chem. Soc. 1952, 74, 3492 .

(5) Dallinga, G.; Mackor, E. L. Rec. Trav. Chim. 1956, 75, 796.

(6) Gaizer, F.; Sipos, P.; Lazar, J. Polyhedron 1994, 13, 855.

(7) See http://www.vitrobot.com/.

(8) Gaponenko, S. V. Optical properties of semiconductor nanocrystals; Cambridge University Press: Cambridge, U.K., 1998.

(9) Papavassilou, G. C.; Mousdis, G. A.; Terzis, A.; Raptopoulou, C. P. Z. Naturforsch. 1999, 54b, 109.

(10) Kodaira, T.; Ikeda, T. Mol. Cryst. Liq. Cryst. 2000, 341, 441.

(11) Landold-Börnstein, Vol. III-17b; Springer-Verlag: Berlin, 1988; pp 297-302.

(12) Nielsen, A. E.; Sohnel, O. J. Cryst. Growth 1971, 11, 233.

(13) Israelachvili, J. Intermolecular \& Surface Forces, 2nd ed.; Academic Press: San Diego, 1992.

(14) Overbeek, J. T. G. Faraday Discuss. Chem. Soc. 1978, 65, 7

(15) Kegel, W. K.; Overbeek, J. T. G.; Lekkerkerker, H. N. W. In Microemulsions, Fundamental and Applied Aspects; Kumar, P., Mittal, K. L., Eds.; Marcel Dekker: New York, 1999; p 13.

(16) Tytko, K.-H.; Glemser, O. Adv. Inorg. Chem. Radiochem. 1976, $19,239$.

(17) Vayssieres, L.; Chaneac, C.; Tronc, E.; Jolivet, J. P. J. Colloid Interface Sci. 1998, 205, 205.

(18) Leff, D. V.; Ohara, P. C.; Heath, J. R.; Gelbart, W. M. J. Phys. Chem. 1995, 99, 7036 .

(19) Mulvaney, P. Colloids Surf., A 1993, 81, 231

(20) Berry, C. Phys. Rev. 1967, 161, 848. 